\section{Supplementary Eye Fields}

M A Sommer, University of Pittsburgh, Pittsburgh, PA, USA

(c) 2009 Elsevier Ltd. All rights reserved.

The supplementary eye fields (SEFs) were discovered in 1985 by John Schlag and Madeleine Schlag-Rey, who were informed by prior findings spanning the preceding century. In 1885, David Ferrier demonstrated that frontal cortex near the midline in monkeys is electrically excitable. By stimulating there, Ferrier evoked movements of the head and eyes toward contralateral space. Homologous sites in the human brain were reported by the neurosurgeons Foerster and Penfield. A breakthrough was provided by Woolsey, who carefully mapped the supplementary motor area in many animals and showed that it had a regular topography that included rostral eye representation. Brinkman and Porter qualitatively explored this zone in 1979. Unlike all of the prior studies, Schlag and Schlag-Rey explored the region in a highly quantitative manner using accurate and precise eye movement measurements. After their seminal work, studies on the function of SEF went in surprising directions.

Early work on the SEF first tested the hypothesis that it is important for generating eye movements, particularly the fast ones known as saccades. This hypothesis quickly fell by the wayside. In its place, other intriguing ideas about SEF function have been promoted and supported. It now appears that the SEF is unnecessary for generating eye movements but is important for higher-order control of eye movement behavior. The SEF might be likened to the foreman of a construction crew for assembling behavior; the SEF does not do the work but guides it.

The unveiling of the SEF as a distinct region in 1985 had special impact due to another landmark set of results published the same year by Bruce and Goldberg that formally delineated the nearby frontal eye field (FEF). Since then, a useful tactic in the field has been to analyze the SEF in relation to its historical twin, the FEF. The present article follows this comparative approach.

\section{Anatomy}

The SEF is located in dorsomedial frontal cortex (Figure 1(a)) and represents the ocular extension of the body map in the supplementary motor area (Figure 1(b)). The exact boundaries of the SEF have been a subject of debate. The functionally defined SEF corresponds roughly to a zone (F7) delineated by Matelli, Luppino, and Rizzolatti using cytoarchitectonics, but associating functional regions with cytoarchitectonic zones is not always straightforward. The FEF, for example, straddles two distinct cytoarchitectonic zones (Walker's 8A and 45) even though its functions seem continuous across them. It is generally accepted that eye-movement-related neurons can be found throughout a large swath of peri-midline cortex, leading some laboratories to select this entire region as their focus of study (using the term dorsomedial frontal cortex rather than SEF). Other laboratories are guided by the principle of studying the zone where eye movements can be elicited with least current threshold using trains of electrical current pulses. In the FEF, a criterion of $<50 \mu \mathrm{A}$ was established, and in an effort to make a fair comparison between SEF and FEF the same criterion was transferred to SEF. The location of SEF in humans is homologous to that in monkeys - in dorsomedial cortex at the rostral end of the supplementary motor area. In terms of connections, the SEF is very similar to the FEF. Both receive projections from, and send efferents to, a plethora of visual- and saccade-related areas with minor differences, such as slightly different patterns of thalamic connections. Functionally, however, the SEF seems more distantly connected than the FEF to the final saccade-generating circuits because the ability to evoke saccades from the SEF requires, in general, relatively higher currents, longer train durations, lower frequencies, and more care in minimizing behavioral obstacles such as intense fixation.

\section{Function}

\section{Generating Saccades and Fixations}

Schlag and Schlag-Rey proposed that the SEF was a distinct eye field because stimulating it elicited saccades and recording from it revealed vision- and saccade-related activity. Follow-up studies established that it also contains many fixation-related cells. Hence, the first hypothesis about SEF was a simple one: perhaps it helps to generate saccades and fixations. At the outset, however, this hypothesis seemed doubtful. A lesion study by Schiller had shown already that when other major oculomotor structures were ablated (the FEF and superior colliculi), animals were permanently devastated in generating saccades even though the SEF remained intact. It may have been that the SEF was indirectly damaged in that study, or that the SEF is important but needs the FEF or superior colliculus to transfer its signals downstream. Later experiments seemed to refute these possibilities, however. Stimulation in the 


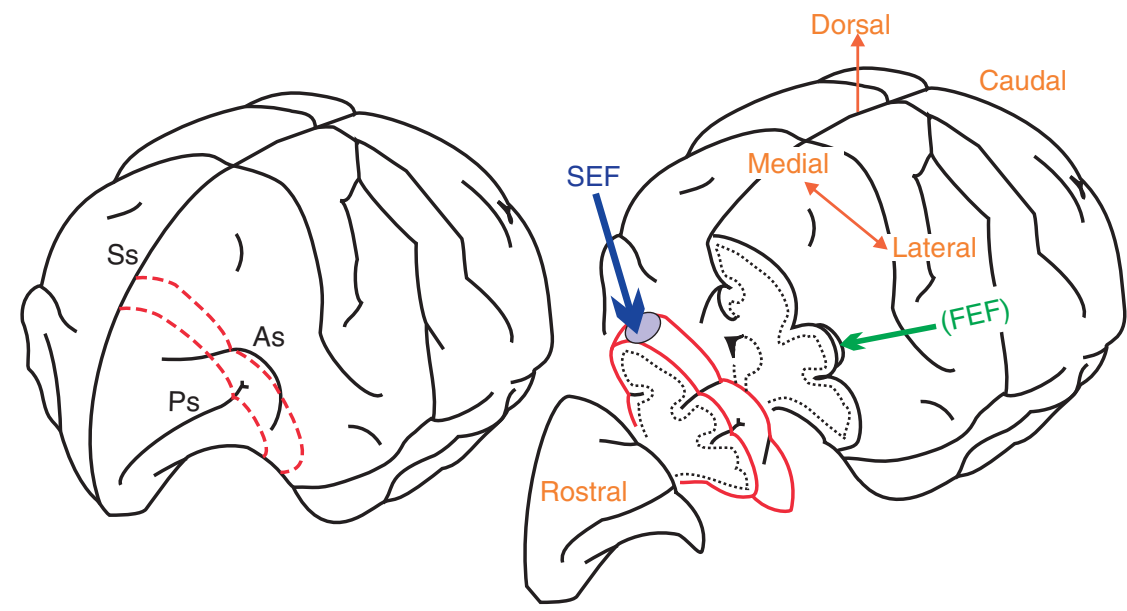

a
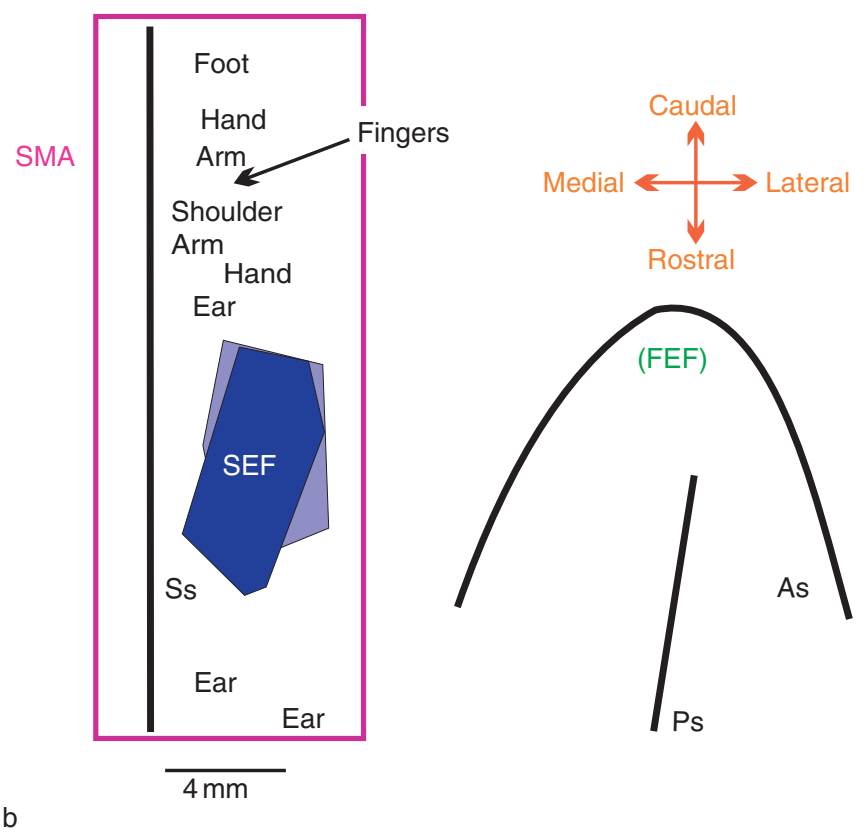

Figure 1 Location of the SEF. (a) Frontal, lateral perspective of monkey brain with general location of SEF and FEF shown in cutaway view at right. As, arcuate sulcus; Ps, principal sulcus; Ss, sagittal sinus. Anatomical directions are shown in orange. (b) Range of SEF recording and stimulation sites (blue) as reported by Schlag and Schlag-Rey. Data from two hemispheres (the two polygons) are superimposed. The SEF lies in the rostral supplementary motor area (SMA), in the head part of the body map. SEF and SMA are functional terms; the anatomical term for the region is the dorsomedial frontal cortex. Location of the FEF is shown for reference to the right. (a) Adapted with kind permission of Springer Science and Business Media from Experimental Brain Research 58, 1985, pp. 208-211, 'Unit activity related to spontaneous saccades in frontal dorsomedial cortex of monkey,' Schlag and Schlag-Rey, fig. 1, (c) Springer-Verlag 1985. (b) Modified and used with permission from Journal of Neurophysiology (Schlag J and Schlag-Rey M (1987) Evidence for a supplementary eye field. Journal of Neurophysiology 57: 179-200).

SEF evokes saccades after lesions of FEF or superior colliculus, implying that the SEF independently accesses saccadic-generating circuits. More to the point, reversible inactivation of the SEF has no discernible effect on saccades and fixations (even though inactivation of the FEF or superior colliculus causes enormous deficits). A study of SEF lesions by the Schiller group found deficits only in an extremely sensitive test of visuosaccadic behavior. The impairments were weak and short-lasting. It is safe to say that the SEF has little or no influence on the basic generation of saccades and fixations.

Stimulating the SEF also elicits head movements and smooth eye movements. No studies have silenced the SEF to test whether it is needed for these actions.

\section{Providing Frames of Reference}

If the SEF is inconsequential for fundamental oculomotor behaviors, then why is it so packed with visual- and eye-related neurons? Why does electrical 
stimulation of it evoke saccades and fixations? These have been the salient questions driving SEF research in the past decade. The mystery is whether the SEF does something important even if it is unconcerned with the details of making individual movements.

From the first studies of Schlag and Schlag-Rey it was recognized that the SEF might play a special role in providing frames of reference. Electrical stimulation of the FEF, superior colliculus, and most other eye movement areas evoke vectors of saccades (i.e., constant displacements regardless of initial eye position). Schlag and Schlag-Rey showed that stimulation of the SEF, in contrast, may evoke goaldirected saccades. Such movements travel to a 'termination zone' in space that stays constant regardless of where the eyes initially fixate (Figure 2(a)). A debate ensued between Tehovnik and colleagues, who confirmed the goal-directed findings and extended them by describing a topographic map of termination zones across the SEF and surrounding dorsomedial frontal cortex, and Bruce and colleagues, who explained the appearance of goal-directedness as natural variation, whereas vector saccades were the norm (Figure 2(b)). The topographic stimulation map of Tehovnik was matched by a topographic map of tuning in SEF fixation-related neurons; similarly, the vector coding of Bruce was matched by the vector coding of SEF saccade-related neurons. It is possible that Tehovnik preferentially activated fixation neurons and Bruce saccade neurons, but this is speculative. On a related note, two laboratories (the Crawford and Schlag groups) showed that some SEF sites produce vector saccades and other SEF sites goaldirected saccades when stimulated, and that vectorto-goal-directed representations in SEF may be organized in a rostral-caudal gradient. The debate about coordinate frames in the SEF has spilled over into a nearly identical controversy regarding whether goal-directed or more limited movements are encoded in primary motor cortex.

A goal-directed or craniotopic (referenced to the head) representation is not the only type of nonstandard coordinate frame encoded by the SEF. Single neuron recording studies by Olson and colleagues found that many SEF neurons use an object-centered frame of reference (Figure 2(c)). Such neurons fire preferentially for saccades made to a particular part of an image (e.g., the left end of a small bar) regardless of where that image is presented. As argued by Olson, object-directed frames of reference are especially critical for us in everyday life as we survey, handle, and inspect the items that surround us. It is unknown to what extent object-directed frames of reference are unique to the SEF.
The bottom line is that there is broad support that the SEF plays a special role in representing frames of reference that are not directly extractable from the retinal image but which require further information: the location of eye in head for craniotopic frames of reference and the segregation of image clutter into distinct entities for object-centered frames of reference.

\section{Supervising Behavior}

The paradox of the SEF - that it seems so saccade related but is so blatantly unnecessary for generating saccades - and the early findings on multiple coordinate systems in the SEF inspired a new generation of experiments that have been very informative. This nascent research posits a higher level function for the SEF. The premise is that the SEF is well poised for controlling saccadic behavior due to its variety of neuronal signals as revealed by recording, its tight linkage to other saccadic centers as revealed by stimulation, and its fluency in multiple ways of spatially encoding visual stimuli and eye movements. The first fundamental question about an executive role for SEF logically would be the following: Can the SEF turn on and off saccades, even if it cannot generate them by itself?

Schall and colleagues studied this question using a task in which a monkey is instructed to make a saccade but then, randomly, may be instructed to cancel it. Nimble supervision of the saccadic system is necessary in this 'countermanding' task, and SEF neurons carry signals that may reflect this supervision. When errors of commission are made (saccades are made when they should have been canceled), many SEF neurons burst vigorously just after the movement (Figure 3(a)). Neurons in FEF, in contrast, seem to have a more proactive function: their activity is well suited to cause saccade generation or cancellation but is poor at representing task outcome.

Whether the error-related activity in SEF influences behavior cannot be answered by neuronal recordings and requires, instead, causal experiments. Schall and Stuphorn performed such a study, electrically stimulating the SEF in the countermanding task. In support of the role of SEF in supervising behavior, activating it with low currents lengthened saccadic reaction times in the countermanding task so that monkeys had more time to cancel their movements (Figure 3(b)). Outside of the countermanding task, however, in a visually guided saccade task, reaction times were made shorter instead of longer. This combination of neuronal recording and stimulation provides complementary evidence for a supervisory role of SEF in controlling whether a saccade is made. 

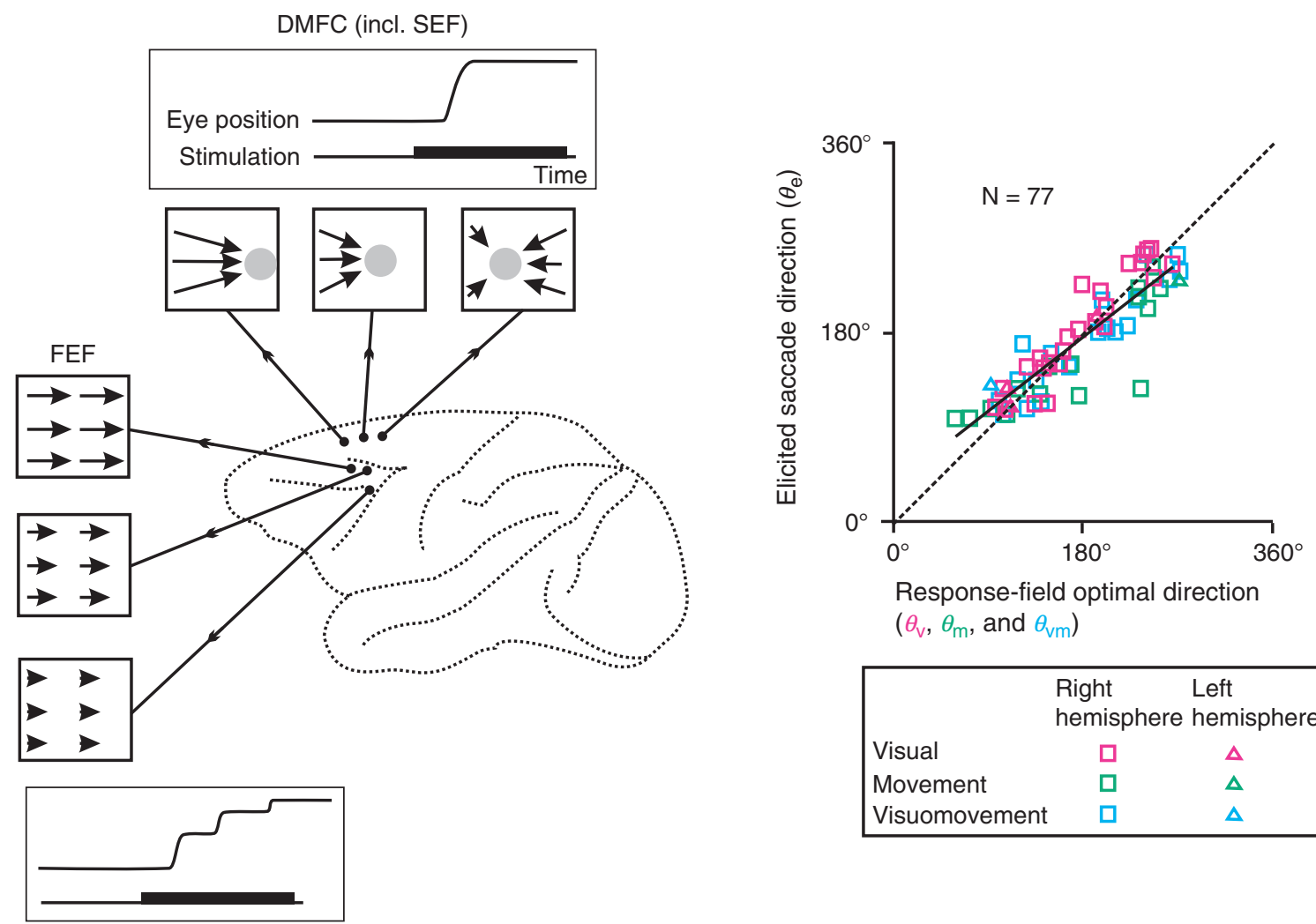

\begin{tabular}{|lcc|}
\hline & Right & Left \\
& hemisphere & hemisphere \\
Visual & $\square$ & $\Delta$ \\
Movement & $\square$ & $\Delta$ \\
Visuomovement & $\square$ & $\Delta$ \\
\hline
\end{tabular}

a

b
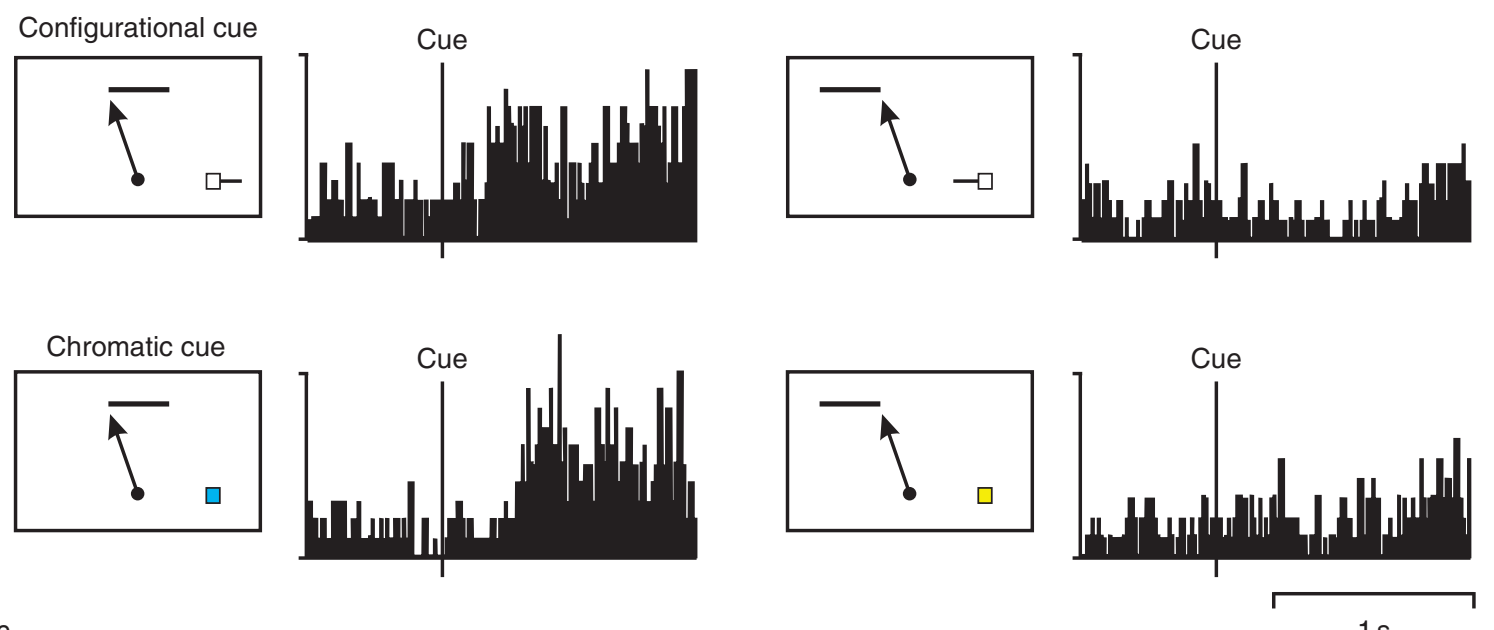

C

Figure 2 Reference frames in the SEF. (a) Head-centered coordinate frame. Saccades (arrows) evoked by stimulation of the dorsomedial frontal cortex (DMFC) including the SEF (top) compared with saccades evoked from the FEF (left). In the DMFC, stimulation-evoked saccades go to a termination zone (gray circle) as a function of stimulated site. Long durations of stimulation cause a single saccade (inset). In FEF, stimulation-evoked saccades exhibit a constant displacement regardless of initial eye position. Long durations of stimulation cause similar saccades to occur repeatedly. (b) Eye-centered coordinate frame. In the core of the SEF, brief train durations evoke directions of saccades ( $y$-axis) that match the directional tuning ( $x$-axis) of visual, movement, or visuomovement neurons found at the stimulated site. Little evidence for termination zones was found in this experiment. (c) Object-centered coordinate frame. Activity of an SEF neuron is strong for saccades made to the left side of a bar (left) but not the right side (right), regardless of the instruction that cues the monkey (square on bar, top; color, bottom). (a) Adapted and reprinted from Behavioral Research Reviews, vol. 32, Tehovnik et al., 'Eye fields in the frontal lobes of primates,' pp. 413-448, ( $) 2000$, with permission from Elsevier. (b) Modified and used with permission from Journal of Neurophysiology (Russo \& Bruce, 84: 2605-2621, 2000). (c) Modified and used with permission from Journal of Neurophysiology (Olson \& Gettner, 81: 2340-2346, 1999). 


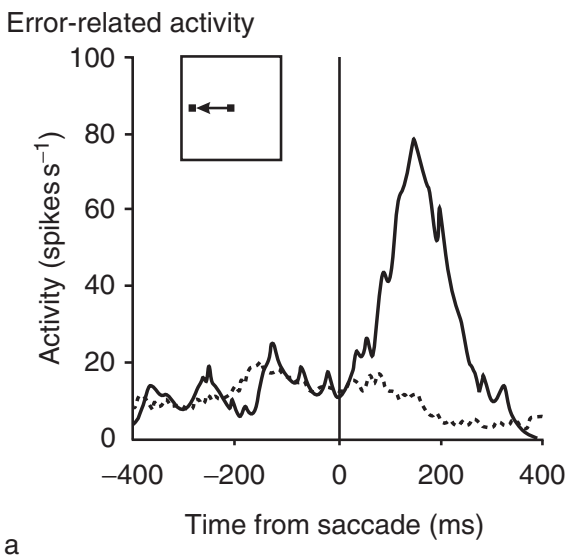

Stimulation-evoked improvement in performance

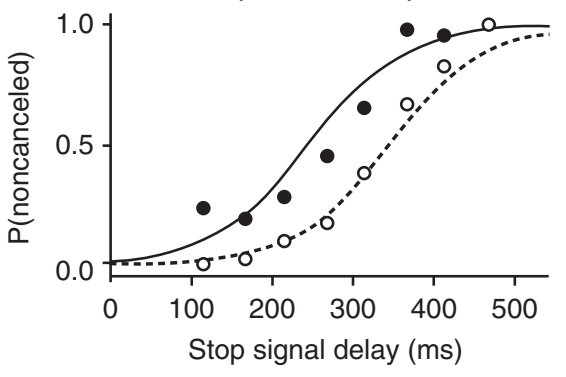

b

Figure 3 Supervisory function of SEF. (a) Average activity of an SEF neuron is shown for saccades made to the left (into ipsilateral space; inset) when the movement was appropriate (dotted line) and when it was errant because it should have been withheld (solid line). The SEF neuron fired vigorously after the error. The same effect occurred for rightward saccades. (b) Improvement in performance when stimulating the SEF (white circles) relative to not stimulating (black circles). Stimulation allowed the monkey to wait even longer - that is, nearer to the last possible moment (rightward shift to longer stop signal delays) before canceling the movement. (a) Adapted by permission from Macmillan Publishers Ltd: Nature 408: 857-860, (c) 2000. (b) Adapted by permission from Macmillan Publishers Ltd: Nature Neuroscience 9: 925-931, (c) 2006 .

\section{Signaling Conflict}

An effective foreman not only gives instructions but also makes wise judgments in the face of conflicting information. So it is with the saccadic system. The visual world provides little information about where to look next, and the signals that are available can provide mixed messages. A traffic light 'walk' signal is good to notice for an instant, but its continual illumination (and sudden change to a flashing 'don't walk' signal) provides a conflict for behavior by demanding your attention when you should, instead, maintain a vigilant watch to the left and right. SEF neurons seem to play a special role in dealing with visuosaccadic conflicts so that one looks in the correct direction, not just the attention-grabbing direction.
One form of visuosaccadic conflict was described previously: in the countermanding task, there is a conflict between the sudden need to fixate and the ongoing plan to make a saccade. Indeed, Schall and colleagues found that a second major cell type in SEF, in addition to the error-encoding type described previously, signaled conflict defined as the successful recognition of a stop signal when saccades were inhibited.

A particularly influential study on visuosaccadic conflict was performed by the discoverers of the SEF, Schlag and colleagues. In their task, a visual stimulus appeared in the periphery but then monkeys had to look away from it. The conflict was between the stimulus location and the required saccadic endpoint. SEF neurons had significantly higher activity in such 'antisaccade' trials than in control trials in which they were allowed to look toward the stimulus (Figure 4(a)). In addition, this antisaccade-related activity was higher in the SEF than in the FEF, providing a rare situation in which signals in the SEF are stronger than those in its more lateral neighbor. One problem in interpreting the results was that the higher activity may have been related to monitoring the conflict, to the different rules implicit in making a saccade toward a target versus away from it, or to other more subtle factors.

To tease apart the reason for elevated SEF activity in the antisaccade task, Olson and colleagues created a task in which the color of a cue, regardless of where it appeared in the visual field, informed the monkey of what vector of saccade to make (left, right, up, or down). The cue was placed randomly to the left, right, up, or down relative to initial fixation. The result was that rules were held constant (color-vector association never varied) but conflict changed from trial to trial (saccades might be made toward, away from, or at right angles to the visual stimulus). In another version of the task, the rule changed so that the monkey simply looked at the stimulus wherever it appeared. The end result was that SEF neurons fired more actively when conflict was high (saccades away from, not toward, stimuli) and rules were difficult (saccades determined by stimulus color, not location; Figure 4(b)). This explains the intense activity in antisaccades versus prosaccades, because in the former conflict is higher and the rule is harder.

\section{Predicting Events}

A common type of neuronal activity found throughout the cerebral cortex is predictive or anticipatory signals. The activity of many neurons ramps up if task context implies that an event, such as visual stimulus onset or a cue to move, is imminent. The SEF seems to 

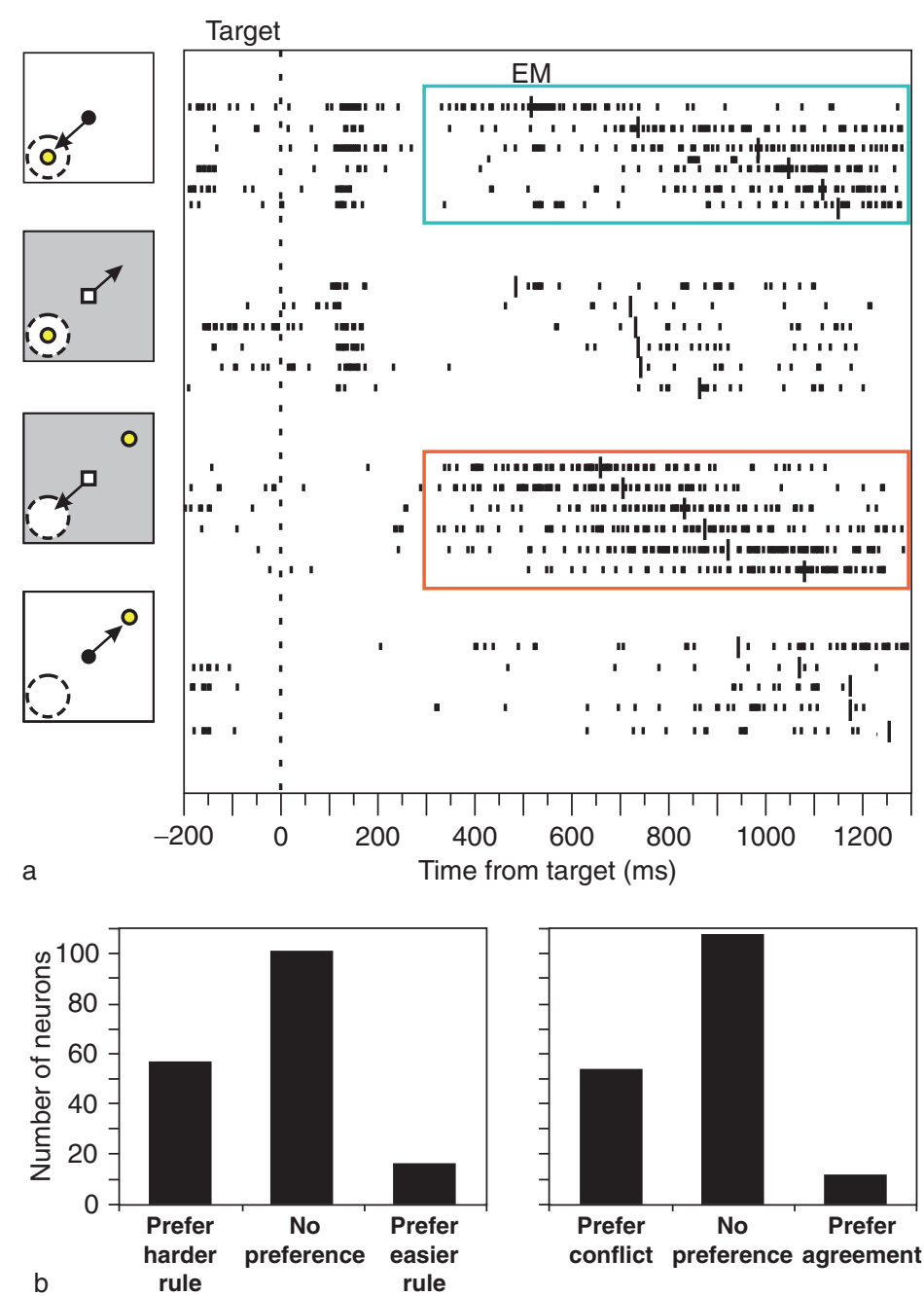

Figure 4 Conflict and rule functions of SEF. (a) SEF activity during antisaccade task. Considering only the trials in which the saccade (arrow) enters the receptive field (dotted circle), the SEF neuron fires more strongly when the saccade is an antisaccade (orange box) than when it is a prosaccade (blue box). The yellow circle shows visual stimulus. Other data show permutations of the task when saccade goes away from the receptive field. (b) The antisaccade task varies from the prosaccade task in that it involves conflict and a harder rule. To tease apart the influence of each factor, conflicts and rules can be varied independently. Both factors are important: SEF neurons fire more strongly for harder rules than easier ones (left) and more strongly for stimulus-response conflict than agreement (right). (a) Adapted by permission from Macmillan Publishers Ltd: Nature 390: 398-401, (c) 1997. (b) Adapted and reprinted from Physiology \& Behavior, vol. 77, Olson and Gettner, 'Neuronal activity related to rule and conflict in macaque supplementary eye field,' pp. 663-670, ( ) 2002, with permission from Elsevier.

be especially predictive in nature, exhibiting more prevalent anticipatory activity than the FEF or an oculomotor region of parietal cortex (the lateral intraparietal area), as shown in a comparative study by the Hikosaka laboratory. The predictive capacity of the SEF was studied in most detail by Heinen and colleagues in relation to smooth pursuit movements. The smooth pursuit system is exquisitely sensitive to regularities in motion and seems to promote prediction avidly. The result is to keep the fovea centered as much as possible on an object of interest rather than having it lag behind due to afferent visual delays. Heinen took advantage of this feature of the smooth pursuit system to quantify the predictive nature of the SEF. In agreement with its other higher-order functions, the SEF was found to be highly active when anticipatory pursuit occurred, and electrical stimulation in SEF promoted anticipatory pursuit. The predictive activity, like the other signals in SEF, seems to be sensitive to rules and other supervisory factors, as shown in a clever task by this group that required monkeys to judge whether a pursuit target would 
cross a 'strike zone'; in this ocular baseball task, anticipatory activity was stronger for strikes than balls. The ability to modulate one's anticipation as a function of context - the point of their findings clearly is important not only for baseball but also for many everyday tasks (e.g., driving a vehicle).

\section{Learning}

Little is known about learning at the neuronal level in the SEF. The only detailed study was performed by Chen and Wise, who demonstrated remarkably strong activity modulations as monkeys learned pairings between foveal-presented stimuli (arbitrary symbols) and direction of saccade required (right, left, up, or down) on a daily basis. For comparison, the monkeys also performed stimulus-saccade pairings that were constant from day to day. One type of neuron seemed to be a 'surprise' detector or learning selective, having activity at the start of learning that disappeared once learning was achieved (Figure 5(a)). Such neurons were not simple error detectors as described by Schall and colleagues because the modulation could occur at any time during the trial - for example, immediately after stimulus onset and not just after an incorrect response. Another type of neuron was learning-dependent, changing its activity in correlation with the learning curve as if mediated by long-term plasticity (Figure $5(b)$ ). Such neurons could in principle store the new stimulus-response association. A striking consequence of their change in activity was that their directional tuning curves shifted during learning. This ties together with the flexibility in spatial coordinate frames represented by SEF neurons, and it raises the question of whether the SEF could represent any coordinate frame with enough training. Whereas learning-related neurons were prevalent in the SEF, they were significantly less common in the FEF.

Learning is a function of many variables, such as detection of stimuli, resolution of conflicts between looking at stimuli and places instructed by stimuli, trial-and-error attempts at finding responses that yield reward, and detection of reward or punishment. The SEF has activity related to sensory input, oculomotor response, conflict resolution, and supervisory control
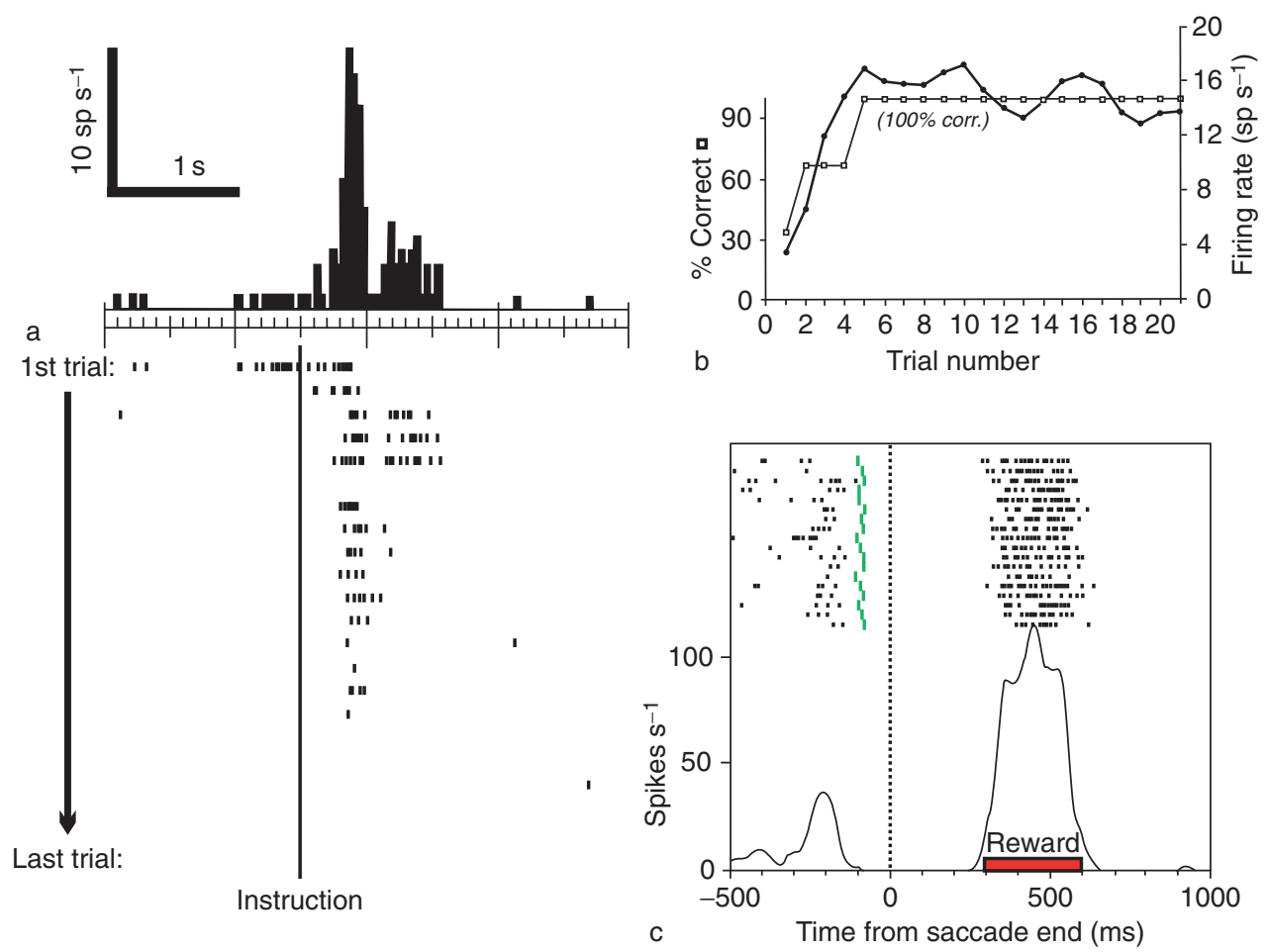

Figure 5 Components of learning in the SEF. (a) For some neurons, activity surges and then disappears as learning progresses (learning-selective neurons). (b) For others, such as the one depicted in this summary graph, activity is correlated with behavioral improvement and then remains at a new level once learning is achieved (learning-dependent neurons). (c) A necessary signal for learning is acknowledgment of reward, and many SEF neurons fire for reward as shown by this example. (a,b) Adapted and used with permission from Journal of Neurophysiology (Chen and Wise, 73: 1101-1121, 1995). (c) Adapted with permission from Journal of Neurophysiology (Amador et al., 84: 2166-2170, 2000). 
of actions. What about reward? Results have been mixed. During a trial, some SEF neurons seem to anticipate reward delivery, but Olson and colleagues suggest that this is uncommon and may be related primarily to motivational state rather than to reward per se. After the behavior necessitated by a trial, and at the time of reward delivery, many SEF neurons do seem to be unambiguously sensitive to detection of reward, as demonstrated by the Schlag laboratory (Figure 5(c)). In summary, the SEF seems to have essentially all of the apparatus necessary to accomplish the learning of visuomotor associations. Still needed are causal experiments to test the necessity of SEF for learning.

\section{Planning Sequences}

The only oculomotor deficit found after reversible inactivation of the SEF and its surrounding cortex has been a mild but significant impairment in performing sequences of saccades, as shown by Sommer and Tehovnik in the Schiller laboratory. In lesion studies, Chou and Schiller demonstrated that damage to the area results in mild, short-term impairments in the ability to process and respond normally to the presentation of multiple visual stimuli. In both studies, saccades to single targets were affected barely or not at all. As with learning, the ability to react correctly when faced with multiple stimuli is the product of many underlying factors. An integrative hypothesis would be that the SEF is necessary for the correct coordination of sequences because of the underlying operations that it performs, such as supervisory control.

An alternative view is that the SEF plays a special role in overseeing the production of sequences. This is supported by further evidence. Hikosaka and colleagues showed that many SEF neurons become strikingly selective for various segments of complex saccadic sequences ('hypersets' of five sequential, learned binary choices). Similar results were found by Tanji and colleagues. Transcranial magnetic stimulation of human SEF reorders saccades of sequences as shown by Tobler and Müri, and Histed and Miller showed that electrical stimulation causes a similar effect in monkeys. In the latter study, reordering of sequences favored a final termination location in contralateral space, thus linking the effect with the coordinate frame results discussed previously.

\section{Coordinating Limbs and Eyes}

By definition, the SEF is an eye field. But how absolute is this eye specificity? The SEF represents the rostral end of a topographic map of the body that extends through the entire supplementary motor area. Edges are blurred everywhere in this map, and it is well documented that skeletomotor representation extends into the oculomotor zone and vice versa. This suggests a supramodal role for the SEF: simply stated, perhaps it is involved in hand-eye coordination. As noted by Tehovnik in a review on the topic, neurosurgeon Penfield and colleagues found that stimulating the area around human SEF caused "the impression that the contralateral hand is raised ... and that meanwhile the gaze is directed by movement of head and eyes toward the hand." By comparing the gross anatomy of where eye-related neurons and armrelated neurons were found in monkey dorsomedial frontal cortex, Tehovnik concluded that overlap is extensive (Figure 6(a)).

Specific studies of eye- and arm-related interactions in neuronal activity were performed by Tanji and colleagues. They had monkeys look at visual targets, reach to them, or look and reach. Exploring the entire dorsomedial frontal cortex, they concluded that eyespecific activity was largely confined to SEF and armspecific activity to more caudal supplementary motor regions, but that there was appreciable overlap (Figure 6(b)). Approximately 7\% of neurons outside of SEF proper were eye-specific, and approximately $14 \%$ of neurons within SEF proper were limbspecific. Other neurons showed complex contingencies (e.g., firing for eye movements if limb movements were withheld but going silent if eye and limb movements co-occurred). In contrast, the FEF was overwhelmingly eye-specific. More work needs to be done to explore multimodality of the SEF and its environs, but the concept that the area plays a special role in hand-eye coordination has received preliminary support.

\section{Conclusion}

The SEF has proven to be one of the more challenging cortical areas to study. Investigators have had to resort to intricate, psychologically based tasks in order to describe SEF activity, but the results have been fruitful. In parallel with the monkey work described here, studies on humans (using metabolic imaging, electroencephalograms, and studies of lesions) have arrived at comparable conclusions regarding human SEF. The human work has been neglected for brevity here, but it is just as important. Much of the progress in understanding the SEF has arisen from systematic application of simple tasks (countermanding and antisaccade tasks) to both the FEF and SEF and comparing the results. Findings from these simple tasks then inspired more complex ones specific to interpreting the functions of the SEF. This slow and steady approach has changed the 


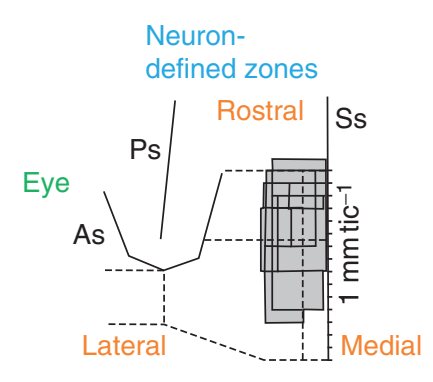

\section{Stimulation-} defined zones
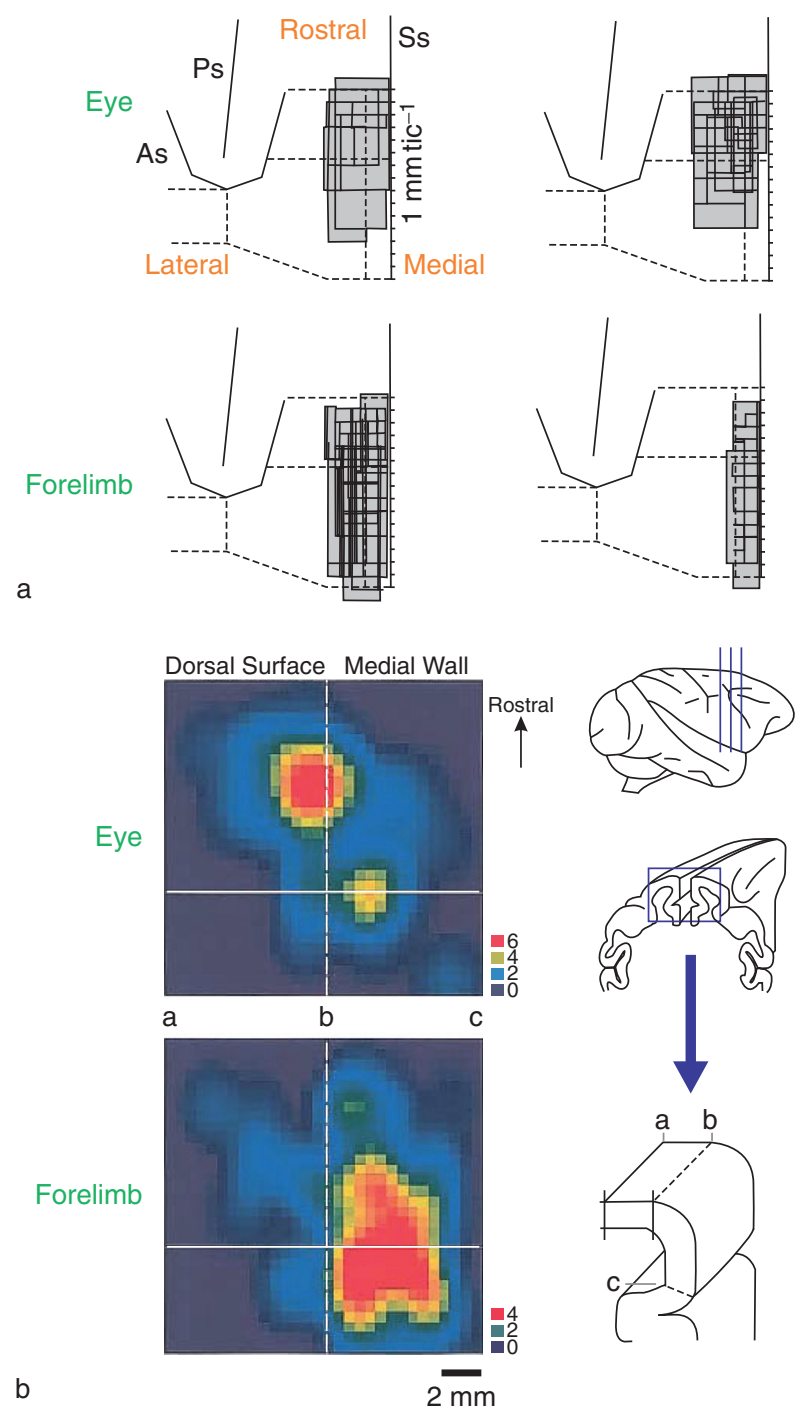

Figure 6 Overlapping limb and eye representation in and around the SEF. (a) Summary maps from a review on eye and forelimb fields in dorsomedial frontal cortex. The left column shows ranges of cortex within which eye-related or forelimbrelated neurons were found in several studies. The right column shows ranges within which eye movements or forelimb movements were evoked by electrical stimulation. There is considerable overlap between the eye and forelimb fields, particularly as defined by neuronal activity. (b) Example map from one study that distinguished eye-related neurons from forelimb-related neurons. Color map (left) represents density of each type of neuron (number of neurons recorded per $\mathrm{mm}^{2}$ ) as a function of cortical space. Orientation and layout of the map is explained by the anatomical diagrams at right. Although there is noticeable segregation, there is overlap as well. (a) Adapted and reprinted from Behavioral Brain Research, vol. 67, Tehovnik, 'The dorsomedial frontal cortex: eye and forelimb fields,' pp. 147-163, (c) 1995, with permission from Elsevier. (b) Adapted and used with permission from Journal of Neurophysiology (Fujii et al., 87: 2158-2166, 2002). general view of the SEF from a frustrating area of study to a rather beautiful, intriguing area. The downside is that hypotheses about SEF function have sprouted wildly. A necessity in future work will be the rigorous application of causal tests, especially reversible inactivation, to establish the functions for which the SEF is truly needed.

See also: Cortical Control of Eye Movements; Frontal Eye Fields; Oculomotor Control: Anatomical Pathways; Spatial Transformations for Eye-Hand Coordination.

\section{Further Reading}

Berman RA, Colby CL, Genovese CR, et al. (1999) Cortical networks subserving pursuit and saccadic eye movements in humans: An fMRI study. Human Brain Mapping 8: 209-225.

Kennard C, Mannan SK, Nachev P, et al. (2005) Cognitive processes in saccade generation. Annals of the New York Academy of Sciences 1039: 176-183.

Lynch JC and Tian JR (2005) Cortico-cortical networks and cortico-subcortical loops for the higher control of eye movements. Progress in Brain Research 151: 461-501.

Martinez-Trujillo JC, Medendorp WP, Wang H, and Crawford JD (2004) Frames of reference for eye-head gaze commands in primate supplementary eye fields. Neuron 44: 1057-1066.

Olson CR (2003) Brain representation of object-centered space in monkeys and humans. Annual Review of Neuroscience 26: 331-354.

Pierrot-Deseilligny C, Rivaud S, Gaymard B, Müri R, and Vermersch AI (1995) Cortical control of saccades. Annals of Neurology 37: 557-567.

Schall JD (1991) Neuronal activity related to visually guided saccadic eye movements in the supplementary motor area of rhesus monkeys. Journal of Neurophysiology 66: 530-558.

Schall JD (1997) Visuomotor areas of the frontal lobe. In: Rockland K, Peters A, and Kaas J (eds.) Extrastriate Cortex of Primates. Cerebral Cortex, vol. 12, pp. 527-638. New York: Plenum.

Schall JD, Stuphorn V, and Brown JW (2002) Monitoring and control of action by the frontal lobes. Neuron 36: 309-322.

Schlag J and Schlag-Rey M (1985) Unit activity related to spontaneous saccades in frontal dorsomedial cortex of monkey. Experimental Brain Research 58: 208-211.

Schlag J and Schlag-Rey M (1987) Evidence for a supplementary eye field. Journal of Neurophysiology 57: 179-200.

Sommer MA and Tehovnik EJ (1999) Reversible inactivation of macaque dorsomedial frontal cortex: Effects on saccades and fixations. Experimental Brain Research 124: 429-446.

Tehovnik EJ (1995) The dorsomedial frontal cortex: Eye and forelimb fields. Behavioural Brain Research 67: 147-163.

Tehovnik EJ and Slocum WM (2004) Behavioural state affects saccades elicited electrically from neocortex. Neuroscience \& Biobehavioral Reviews 28: 13-25.

Tehovnik EJ, Sommer MA, Chou IH, Slocum WM, and Schiller PH (2000) Eye fields in the frontal lobes of primates. Brain Research Reviews 32: 413-448. 